\title{
Bibliografía especializada de Manuel Mora Valverde
}

\author{
Jorge A. Meneses Hernández*
}

Recibido: Marzo 2012 • Aceptado: Junio 2012

\section{RESUMEN}

Don Manuel Mora Valverde se ha distinguido sobre todo como político. Prueba de ello es la presente compilación de referencias bibliográficas de documentos, discursos, artículos de revista, entre otros, escritos por don Manuel o con él como tema de crítica o análisis. Esta bibliografía especializada agrupa los documentos de importancia nacional que se encuentran en las bibliotecas de la Universidad Estatal a Distancia, Universidad de Costa Rica, Universidad Nacional, Biblioteca Nacional y Biblioteca del Congreso de los Estados Unidos. Asimismo, contiene referencias bibliográficas con enlaces a los documentos disponibles en la internet a texto completo. Está dirigida a todas las personas interesadas en estudiar y aprender de su pensamiento a través del tiempo y su contribución a la política nacional.

Palabras clave: Manuel Mora Valverde - bibliografía especializada, bibliografías, historia política - Costa Rica, Partido Comunista - Costa Rica.

\begin{abstract}
Manuel Mora Valverde was an important 20th. Century political leader in Costa Rica. This is a bibliographic compilation of documents, speeches, journal articles and other sources that were written by Mora as single author or as coauthor. The libraries covered are, in Costa Rica, Universidad Estatal a Distancia, Universidad de Costa Rica, Universidad Nacional and Biblioteca Nacional ; and in the USA, the Library of Congress. There are links to those documents that are available online, as a service to researchers and students who want to assess Mora's thought and contribution to Costa Rican politics.
\end{abstract}

Key words: Manuel Mora Valverde - specialized literature, bibliographies, history - politics Costa Rica, Communist Party - Costa Rica.

* Encargado de Cátedra de Tratamiento de la Información, Programa de Bibliotecología, Universidad Estatal a Distancia. E-mail: jmeneses@uned.ac.cr 


\section{Introducción}

La presente bibliografía especializada es una lista descriptiva de documentos que se han publicado del costarricense Manuel Mora Valverde (1909-1994). El objetivo principal de la bibliografía especializada de este destacado costarricense es una guía para el investigador interesado en sus aportes a la historia de Costa Rica.

Se consultaron los catálogos en línea de las bibliotecas de las siguientes instituciones:

- Universidad Estatal a Distancia. Biblioteca Central.

- Universidad de Costa Rica. Sistema de Bibliotecas, Documentación e Información.

- LATINDEX Repositorio de las Revistas Científicas. Universidad de Costa Rica.

- Universidad Nacional. Biblioteca Joaquín García Monge.

- Biblioteca Nacional (Costa Rica).

- Biblioteca del Congreso de los Estados Unidos.

Se han incluido las referencias de los documentos disponibles a texto completo en internet.

Se definió el término Manuel Mora Valverde para la búsqueda de información, seleccionando documentos, tales como: libros, publicaciones periódicas, artículos aparecidos en otras publicaciones en el ámbito nacional e internacional y documentos escritos por el autor. Destacan, además, descriptores tales como: Comunismo - Costa Rica, Comunismo - Centro América, Huelgas - Costa Rica -1948, Picado Michalsky, Teodoro, Calderón Guardia, Rafael Ángel, 1900 - 1970, Costa Rica - Historia - Revolución 1948, Figueres Ferrer, José, Villegas Hoffmeister, Guillermo, que complementan el tema de la bibliografía que se presenta a continuación.

Para el investigador interesado en la consulta de estos documentos, las bibliotecas brindan el servicio de préstamo interbibliotecario nacional o internacional, por un período de tiempo determinado; se solicita la copia impresa o digital del documento como parte del servicio ofrecido.

\section{Biografía}

Se han recogido cientos de palabras de la vida de don Manuel Mora Valverde por sus aportes al país, por el rumbo que este tomó con la fundación del Partido Comunista el 16 de junio de 1937, en el cual participó por más de 50 años. Desde joven Mora Valverde escuchó e interiorizó las palabras del general Volio, que despertaron su vocación política; y en su hogar, por parte de su padre don José 
Aportes de don Manuel Mora

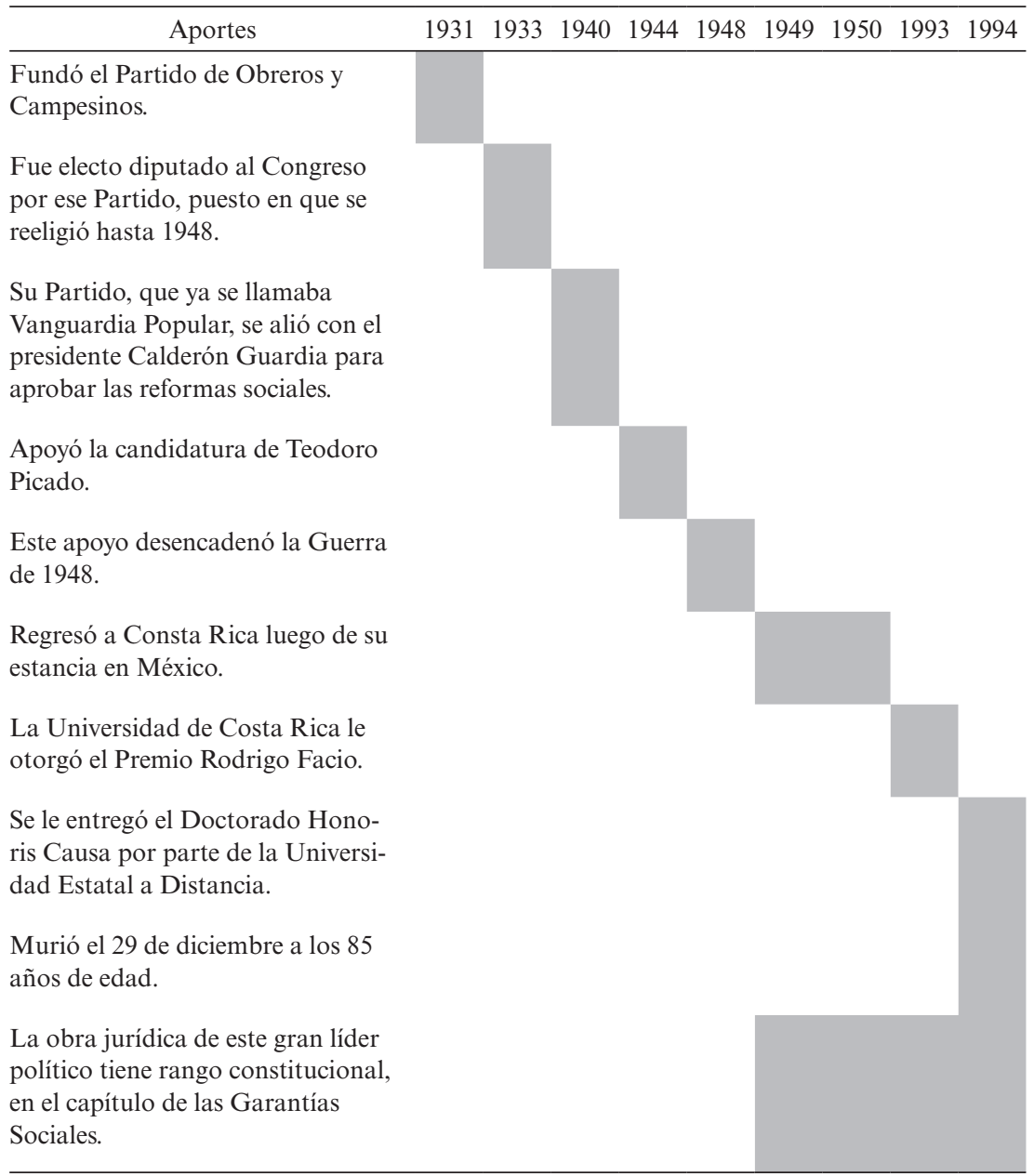

Fuente: Elaboración propia.

Rafael Mora Zúñiga, las teorías marxistas leninistas; la lectura del Manifiesto Comunista se convirtió en el norte del pensamiento de don Manuel.
Sus estudios en Derecho le permitieron colaborar en la defensa del valor de la justicia, ofreciendo un enfoque adecuado del problema que tiene el ciudadano 


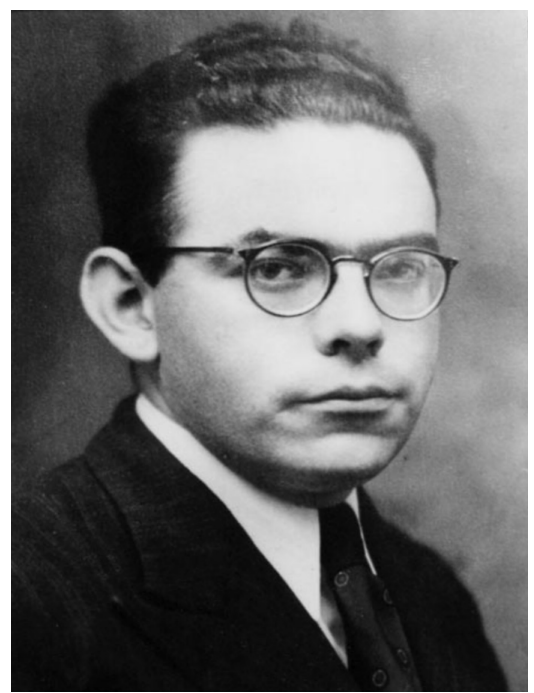

Fuente: Asamblea Legislativa. Benemérito de la Patria: Manuel Mora Valverde. Recuperado de: http://www.asamblea.go.cr/Centro_de_informacion/Sala_Audiovisual/Benemritosdelapatria/ Forms/DispForm.aspx?ID=83

de los sectores sociales y obreros del país. La semblanza de don Manuel Mora en la Asamblea Legislativa cita que "todas ellas (sus actuaciones) estuvieron matizadas siempre con el sello que él les imprimía: un nacionalismo inclaudicable y su lucha sin tregua contra la explotación de los obreros o de la riqueza de la Nación" (Asamblea Legislativa, 2009) ${ }^{1}$.

1. Asamblea Legislativa (2009). Benemérito de la Patria: Manuel Mora Valverde. Recuperado de http://www.asamblea.go.cr/ Centro_de_informacion/Sala-Audiovisual/ Benemeritos $\% 20 \mathrm{de} \% 201 \mathrm{a} \% 20$ patria/Forms/ DispForm.aspx?ID=83

\section{Referencias bibliográficas}

Intercambio de cartas entre el Arzobispo Víctor Sanabria y Manuel Mora Valverde. (14 de Junio de 1943). Recuperado el 10 de Marzo de 2012, de http:// www.worldpolicy.org/sites/default/ files/uploaded/image/Sanabria-Mora\%20Valverde-1943.pdf

El Colegio de Abogados de Costa Rica en recuerdo del colega Manuel Mora Valverde. (1995 feb.). Reflexiones, 31. Disponible en Universidad de Costa Rica. Sistema de Bibliotecas, Documentación e Información, 15-16.

Barahona Rivera, M. (2009). Centenario de Manuel Mora Valverde y los últimos días de la guerra civil. Revista estudios, 22. Disponible en Universidad de Costa Rica. Sistema de Bibliotecas, Documentación e Información, 85-104.

Barahona Rivera, M. (2009). El partido comunista costarricense. Revista estudios 22. Recuperado de http://www.estudiosgenerales.ucr.ac.cr/estudios/no22/ papers/iisec2.html.

Barahona Rivera, M. (2009). El partido comunista costarricense. Revista estudios 22. Recuperado de http://www.estudiosgenerales.ucr.ac.cr/estudios/no22/ papers/iisec2.html.

Castillo López, A. (1995 feb.). Manuel Mora Valverde: Doctor Honoris Causa Universidad Estatal a Distancia. Reflexiones, 31. Disponible en Universidad de Costa Rica. Sistema de Bibliotecas, Documentación e Información, 9-14.

Chacón Araya, G. (2009). Carlos Luis Fallas: militante comunista. Revista comunicación vol. 18. Recuperado de http://redalyc.uaemex.mx/ pdf/166/16611988008.pdf, 47-50. 
Chacón Araya, G. (2009). Carlos Luis Fallas: militante comunista. Revista comunicación vol. 18. Recuperado de http://redalyc.uaemex.mx/pdf/166/ 16611988008.pdf, 47-50.

Chacón, V. (6 de Agosto de 2009). En agosto de 1934 los trabajadores bananeros se alzaron en un movimiento que cambió la historia de la lucha de clases en Costa Rica. Semanario Universidad. Recuperado de: http://www. semanariouniversidad.ucr.cr/index. $\mathrm{php} / \mathrm{component/content/article/369-}$ Cultura/1302-hace-75-anos-se-sangroen-la-huelga-del-atlantico.html.

Contreras Alvarez, G. ([1995?]). Manuel Mora y los logros de la democracia costarricense. San José, C.R.: Impr. Nacional. Disponible en Universidad de Costa Rica. Sistema de Bibliotecas, Documentación e Información.

Contreras, G. (2010). El carácter de las reformas sociales durante la década de los cuarenta del siglo XX en Costa Rica. Revista pilquen no 12 año XXI. Recuperado de http://www.scielo.org. ar/pdf/spilquen/n12/n12a21.pdf.

De la Cruz de Lemos, V. (1995 feb). Manuel Mora Valverde: facetas de su vida y sus luchas. Reflexiones, 31. Disponible en Universidad de Costa Rica. Sistema de Bibliotecas, Documentación e Información, 17-28.

Esquivel Corella, F. (2008). "Cuestión social" y políticas sociales en el estado empresario: reflexiones históricas para el trabajo social. Rev. Reflexiones 87 (1). Recuperado de: http://www.latindex.ucr.ac.cr/reflexiones-87-1/reflexiones-87-1-01.pdf, 9-24.

Lyra, C. (8 de Noviembre de 2011). La CCSS me preocupa. Semanario
Universitario. Recuperado de http:// www.semanariouniversidad.ucr. cr/index.php/component/content/ article/1442-Opini $\%$ C3\%B3n/4806-laccss-me-preocupa.html.

Merino del Río, J. (1996). Manuel Mora y la democracia costarricense: viaje al interior del Partido Comunista. Heredia, C.R: Editorial Fundación UNA. Disponible en Library of Congress.

Merino del Río, J. A. (1996). Manuel Mora y la democracia costarricense: viaje al interior del partido comunista. Heredia, C.R.: Fundación UNA. Disponible en Universidad de Costa Rica. Sistema de Bibliotecas, Documentación e Información.

Molina Jiménez, I. (2009). Los materiales impresos comercializados por el partido comunista de Costa Rica. Una contribucion documental (1931-1948). Rev Ciencias Sociales 123-124. Recuperado de http://163.178.170.74/ wp-content/revistas/123-124/12-MOLINA.pdf, 185-225.

Molina Jiménez, I. (2009). Los materiales impresos comercializados por el partido comunista de Costa Rica. Una contribución documental (19311948). Rev. Ciencias Sociales 123124. Recuperado de: http://www. latindex.ucr.ac.cr/sociales-123-124/sociales-123-124-12.pdf, 185-225.

Mora Rodríguez, A. (5 de Enero de 1995). Manuel Mora y su papel en la historia patria. La República, pág. 17 a.

Mora Rodríguez, A. (s.f.). Manuel Mora y el socialismo "a la tica". Disponible en: Universidad Nacional. Biblioteca Joaquín García Monge .

Mora S, M. (1994 jul). Dr. Manuel Mora Valverde. Reflexiones, 24. Disponible 
en Universidad de Costa Rica. Sistema de Bibliotecas, Documentación e Información, 3-8.

Mora V, E. (1988). De Santino a Stalin. San José, C.R.: Revolución. Disponible en Universidad de Costa Rica. Sistema de Bibliotecas, Documentación e Información.

Mora Valverde, E. (19 de Enero de 1989). En la Penitenciaria Central. Libertad, pág. 12.

Mora Valverde, E. (31 de Enero de 1995). Una vida en lucha por los intereses de la clase trabajadora. La Gaceta. Suplemento, pág. 4 .

Mora Valverde, M. (1928 oct). Discurso del alumno Manuel Mora V. Anales universitarios 1 (7). Disponible en Biblioteca Nacional, 197 - 198.

Mora Valverde, M. (1937). Tres discursos en defensa de la democracia. San José, C.R.: Impr. La Tribuna. Disponible en: Universidad Nacional. Biblioteca Joaquín García Monge.

Mora Valverde, M. (1939). En respuesta a los falsos defensores de la democracia y de la independencia de las pequeñas naciones: dos discursos. San José, C.R.: Comité de Prensa del Partido Comunista de Costa Rica. Disponible en Universidad de Costa Rica. Sistema de Bibliotecas, Documentación e Información.

Mora Valverde, M. (1939). Por la afirmación de nuestra democracia. San José, C.R.: Comité Central del Partido Comunista. Disponible en Library of Congress.

Mora Valverde, M. (1940). Imperialismo: nuestra soberanía frente al Departamento de Estado. San José, C.R.:
Partido Comunista de Costa Rica. Disponible en Library of Congress.

Mora Valverde, M. (1958?). Dos discursos en defensa de Vanguardia Popular. San José, C.R.: Disponible en Biblioteca Nacional.

Mora Valverde, M. (1958?). Dos discursos en defensa de Vanguardia Popular: contestación a Don Otilio Ulate y a Don José Figueres. San José, C.R.: Disponible en Library of Congress.

Mora Valverde, M. (1963). Crisis y revolución. San José, C.R.: Imprenta Elena. Disponible en Biblioteca Nacional.

Mora Valverde, M. (1963). Crisis y revolución. San José, C.R: Imprenta Elena. Disponible en: Universidad Nacional. Biblioteca Joaquín García Monge.

Mora Valverde, M. (1969). Entrevista. (J. Wilkie, \& E. Wilkie, Entrevistadores)

Mora Valverde, M. (1980). Discursos, 19341979. San José, C.R.: Presbere. Disponible en Universidad de Costa Rica. Sistema de Bibliotecas, Documentación e Información.

Mora Valverde, M. (1980). Discursos: 1934 1979. San José, C.R.: Editorial Presbere. Disponible en Biblioteca Nacional.

Mora Valverde, M. (1980). Discursos: 1934 - 1979 / Manuel Mora Valverde; selección, prólogo y notas de Gilberto Calvo, Francisco Zúñiga Díaz. San José, C.R.: Editorial Presbere. Disponible en Library of Congress.

Mora Valverde, M. (1985). Centroamérica, negociación o guerra. San José, C.R.: Editorial Revolución. Disponible en Biblioteca Nacional.

Mora Valverde, M. (1985). Centroamérica: negociación o guerra: la posición 
de los comunistas costarricenses. San José, C.R.: Editorial Revolución. Disponible en Library of Congress.

Mora Valverde, M. (12 de Diciembre de 1986). A los militantes del Partido del Pueblo. Libertad, pág. 11 Disponible en Biblioteca Nacional.

Mora Valverde, M. (17 de Octubre de 1986). Avance positivo hacia desarme. Libertad, pág. 2 Disponible en Biblioteca Nacional.

Mora Valverde, M. (24 de Octubre de 1986). El plan que se cocina contra la paz de Costa Rica. Libertad, pág. 2 Disponible en Biblioteca Nacional.

Mora Valverde, M. (5 de Diciembre de 1986). Impedimos que Costa Rica fuera ocupada por los EEUU y Somoza y salvamos todas las leyes sociales conquistadas con sangre obrera. Libertad, pág. 3 Disponible en Biblioteca Nacional.

Mora Valverde, M. (30 de Enero de 1987). El Pacto de Ochomogo impidió ocupación militar en Costa Rica. Libertad, pág. 2.

Mora Valverde, M. (6 de Noviembre de 1987). Manuel Mora aborda el serio problema del Caribe. Libertad, pág. 2 Disponible en Biblioteca Nacional.

Mora Valverde, M. (6 de Noviembre de 1987). Manuel Mora aborda el serio problema del Caribe. Libertad, pág. 2.

Mora Valverde, M. (14 de Agosto de 1987). Manuel Mora comenta los resultados de la cumbre de Guatemala. Libertad, pág. 2 Disponible en Biblioteca Nacional.

Mora Valverde, M. (27 de Noviembre de 1987). Manuel Mora comienza a explicar sobre las elecciones de 1948. Libertad, pág. 2 Disponible en Biblioteca Nacional.

Mora Valverde, M. (11 de Diciembre de 1987). Manuel Mora explica la huelga de brazos caídos y la nulidad de las elecciones de 1948. Libertad, pág. 2. Disponible en Biblioteca Nacional.

Mora Valverde, M. (27 de Febrero de 1987). Manuel Mora relata entretelones de la guerra civil. Libertad, pág. 2 Disponible en Biblioteca Nacional .

Mora Valverde, M. (21 de Agosto de 1987). Manuel Mora Valverde comenta los resultados de la Cumbre de Guatemala. La Nación, pág. 24.

Mora Valverde, M. (15 de Enero de 1988). Esquipulas III. Libertad, pág. 2. Disponible en Biblioteca Nacional.

Mora Valverde, M. (3 de Marzo de 1988). La deuda externa de América Latina. Libertad, pág. 4. Disponible en Biblioteca Nacional.

Mora Valverde, M. (16 de Junio de 1988). Lo que procede es que la energía eléctrica la controle directamente el Estado. Libertad, págs. 8-9. Disponible en Biblioteca Nacional.

Mora Valverde, M. (26 de Marzo de 1990). El código de trabajo y la realidad. $\mathrm{La}$ Prensa Libre, pág. 11 Disponible en Biblioteca Nacional.

Mora Valverde, M. (11 de Julio de 1993). Los comunistas sí contribuimos. La República, pág. 18 A. Disponible en Biblioteca Nacional.

Mora Valverde, M. (1995 feb). Discurso del 12 de junio de 1934. Reflexiones, 31. Disponible en Universidad de Costa 
Rica. Sistema de Bibliotecas, Documentación e Información, 29-47.

Mora Valverde, M. (200?). Ensayo: dos discursos / del licenciado Manuel Mora Valverde; comentado por Merino Ramírez Huertas. San José, C.R.: Disponible en Library of Congress.

Núñez, B. (19 de Abril de 1948). Carta del Pbro Núñez al Licdo. Mora Valverde. Recuperado el 10 de Marzo de 2012, de http://fundacionenriquebolanos. org/FB_RC/RC_1964_01_N40/files/ assets/downloads/page0108.pdf

Ramírez, V. (1980). Pacto Ochomogo. (Teletica Canal 7, Entrevistador)

Rivera, E. (1 de Junio de 2012). Comunistas festejaron 80 años reafirmando su vocación marxista. La Nación. Recuperado de: http://www.nacion. com/2011-06-19/ElPais/comunistasfestejaron-80-anos-reafirmando-suvocacion-marxista-.aspx.

Rojas Bolaños, M. (1978). El desarrollo del movimiento obrero en Costa Rica: un intento de periodización. Cien Soc 15-16: 13-31. Recuperado de http://163.178.170.74/wp-content/revistas/15-16/rojas.pdf, 13-30.

Rovira Mas, J. (2007). Gerardo Contreras Álvarez y el 75 aniversario de la Fundación del Partido Comunista de Costa Rica. Rev Ciencias Sociales 115. Recuperado de http://www.latindex. ucr.ac.cr/sociales-115/10-rovira.pdf, 137-141.

Salas, A. (1997). Con Manuel, devolver al pueblo su fuerza. San José, C.R.: Editorial de la Universidad de Costa Rica. Disponible en Biblioteca Nacional.
Salas, A. (1997). Con Manuel: devolver al pueblo su fuerza. San José, C.R.: Edit. de la UCR. Disponible en Universidad de Costa Rica. Sistema de Bibliotecas, Documentación e Información.

Salguero, M. (1981). Tres meses con la vida de un hilo: crónicas y entrevistas (Manuel Mora - José Figueres). San José, C.R.: Editorial Universidad Estatal a Distancia. Disponible en Library of Congress.

Salguero, M. (11981). Tres meses con la vida en un hilo: crónicas y entrevistas. San José, C.R.: EUNED. Disponible en Universidad Estatal a Distancia. Biblioteca Central.

Salguero, M. (1981). Tres meses con la vida en un hijo: crónicas y entrevistas, Manuel Mora - José Figueres. San José, C.R.: Editorial Costa Rica. Disponible en Biblioteca Nacional.

Salom Echeverría, R. (1995 dic.). Manuel Mora: in memoriam. Reflexiones 41. Disponible en Universidad de Costa Rica. Sistema de Bibliotecas, Documentación e Información, 13-16.

Salguero, M. (1985). Tres meses con la vida en un hilo : crónicas y entrevista (Manuel Mora - José Figueres). San José, C.R.: Edit. Costa Rica. Disponible en Universidad de Costa Rica. Sistema de Bibliotecas, Documentación e Información.

Solís, M. A. (2007 - 2008). El 48 como desorden trágico. Anuario de Estudios Centroamericanos, Universidad de Costa Rica, 33-34. Recuperado de: http:// www.latindex.ucr.ac.cr/anuario-33-34/ anca005-09.pdf, 261-295. 
Universidad de Costa Rica. Facultad de Ciencias Sociales. (1995 feb.). Lic. Manuel Mora Valverde: premio Rodrigo Facio 1992: Universidad de Costa Rica. Reflexiones 31. Disponible en Universidad de Costa Rica. Sistema de Bibliotecas, Documentación e Información, 3-7.
Universidad Estatal a Distancia. (2009). Periódico La Revolución. San José, C.R.: EUNED. Disponible en Universidad Estatal a Distancia. Biblioteca Central.

Vásquez Vargas, M. (2007). Búsqueda de la libertad en dos textos de Fabián Dobles. Káñina, Rev. Artes y Letras, Univ. Costa Rica. Vol. XXXI (2) Recuperado de: http://www.latindex.ucr. ac.cr/kanina-31-2/23-VASQUEZ.pdf, 273-280. 
